\title{
Helminth communities and host- parasite relationships in argentine brown rat (Rattus norvegicus)
}

\author{
I. E. GOMEZ VILLAFAÑE ${ }^{1,2}$, M. R. ROBLES ${ }^{2,3}$, M. BUSCH ${ }^{1,2}$
}

\begin{abstract}
${ }^{1}$ Laboratorio de Ecología de Poblaciones. Facultad de Ciencias Exactas y Naturales, Universidad de Buenos Aires, Buenos Aires, Argentina, E-mail: isabelgv@ege.fcen.uba.ar; ${ }^{2}$ Consejo Nacional de Investigaciones Científicas y Tecnicas, Buenos Aires, Argentina; ${ }^{3}$ Centro de Estudios Parasitológicos y de Vectores CEPAVE (CCT- CONICETLa Plata) (UNLP), Calle $2 \mathrm{~N}^{\circ}$ 584, 1900 La Plata, Buenos Aires, Argentina
\end{abstract}

\begin{abstract}
Summary
The aims of this research were: 1) to determine the helminth parasite fauna of seventy two Rattus norvegicus (Berkenhout, 1769) individuals inhabiting poultry farms in the Pampean region of Argentina; 2) to examine the relationship between parasitism and individual characteristics of the hosts, and 3) to analyze the associations among helminths. The study was carried out in twenty-four poultry farms from autumn 2000 to winter 2001. Nematodes were found in the intestine or caecum rectum of the $74 \%$ of rats and cestodes were found along all the intestine of the $28 \%$ of rats. Heterakis spumosa (Travassos 1914) was a central species, whereas Syphacia muris, Yamaguti 1941, Nippostrongylus brasiliensis (Travassos 1914) and Hymenolepis diminuta (Rudolphi 1819) were satellite species. Helminths do not affect rat survival and growth of rats. Rats parasitized with $H$. spumosa and $H$. diminuta were larger and older than non-parasitized rats. The intensity of infection with $H$. spumosa significantly increased with rat age. Rats parasitized with Nippostrongylus brasiliensis and Syphacia muris did not differ in size and age with respect to non-parasitized ones. The mean intensity of infections per host was 33.74, 74.28, higher than 200, and 3.10 for $H$. spumosa, $S$. muris, $N$. brasiliensis and $H$. diminuta, respectively. The mean intensity of infection with $H$. spumosa was higher in summer than in the other seasons, while the higher mean intensity of infection with $N$. brasiliensis and $S$. muris occurred in autumn. Infections with Heterakis spumosa and Syphacia muris, and with Syphacia muris and Nippostrongylus brasiliensis were independent; while Heterakis spumosa and Nippostrongylus brasiliensis were negatively associated.
\end{abstract}

Key words: helminths; Heterakis spumosa; Hymenolepis diminuta; Nippostrongylus brasiliensis; Rattus norvegicus; Syphacia muris

\section{Introduction}

Parasitic infections are one of the factors that influences population dynamics, not only directly by affecting reproductive or survival parameters of one species, but also indirectly by changing both intra and interspecific interactions, decreasing competitive abilities or increasing vulnerability to predators. Although parasites rarely kill their hosts, they can produce a reduction in body mass, size and/or a change in behavior that ultimately may affect reproduction or survival of them. To evaluate the role of parasitism on the population dynamics of a species, is necessary to study both populations, the host and the parasite (Wisnivesky, 2003).

Helminth species such as Hymenolepis diminuta (Rudolphi 1819); Taenia taeniaeformis (Batsch 1786); Capillaria hepatica (Bancroft 1893); Syphacia muris Yamaguti 1941; Nippostrongylus brasiliensis (Travassos 1914); Heterakis spumosa Schneider, 1866 and Toxocara spp. are associated to Rattus norvegicus (Berkenhout, 1769). These helminths have been cited in numerous studies, but these consist of descriptions, lists and new records of hosts and localities, and do not provide data about the relationship between the helminths and the individual characteristics of their hosts (Seong et al., 1995; Cabrera \& Mendoza, 2001; Pinto et al.; 2001; Iannacone \& Alvariño, 2002, Stojcevic et al., 2004). However, the presence and the intensity of the parasites are related with the characteristics of the host, the interaction among the parasites (intraspecific and interspecific competition), and with the external environment (Iannacone \& Alvariño, 2002; Stojcevic et al., 2004). On other hand, of the surveys that were carried out of rodents, there have been only limited reports on intestinal parasites of rats (Suriano \& Navone, 1992, 1994; Seong et al., 1995; Cabrera \& Mendoza, 2001; Pinto et al., 2001; Iannacone \& Alvariño, 2002).

The aims of this work were: 1) to determine the helminth parasite fauna of $R$. norvegicus individuals that inhabiting poultry farms in the Pampean region of Argentina; 2) to 
examine the relationship between parasitism and individual characteristics of the hosts, and 3) to analyze the associations among helminths.

\section{Materials and methods}

The study was carried out in Exaltación de la Cruz, Buenos Aires Province, Argentina, $34^{\circ} \mathrm{S}, 59^{\circ} \mathrm{W}$. The study area is located in the Pampa Ondulada and has a temperate climate. Mean annual temperature is $16{ }^{\circ} \mathrm{C}$, and annual rainfall averages $1000 \mathrm{~mm}$. Rat samplings were conducted from autumn 2000 to winter 2001 in twenty-four poultry farms random selected among fifty-five poultry farms of the study area. Rats were captured in live traps of $15 \mathrm{x} 16 \mathrm{x}$ $31 \mathrm{~cm}$. Traps were baited with meat and carrot, and were active for three consecutive nights per farm and season. We studied six farms per season of each year. For each rat captured we recorded species according with external and cranial morphology, body mass (in $\mathrm{g}$ ), size (in $\mathrm{cm}$ ) and age (months). The age was estimated on based of the dry weight of the eye lens (Kataranovski et al., 1994). All individuals were manipulated according with the norms of the National law $\mathrm{N}^{\circ} 14.346$ of Argentina.

We examined three portions of equal length of the intestine (upper intestine, medium intestine and lower intestine), and the caecum-rectum for the presence of helminths. Helminths were fixed in alcohol $70^{\circ}$, cleared with lactophenol and observed under a microscope. The cestodes collected were stained in acetic carmin, dehydrated in a series of alcohols and mounted in Canada balsam. For the identification to the generic level we used the taxonomic key of Khalil et al. (1994) and Anderson et al. (1974). Voucher specimens were deposited in the Helminthological Collection of Museo de La Plata (H. spumosa 5579, S. muris 5580, N. brasiliensis 5872, H. diminuta 5871), and their host, $R$. norvegicus, were deposited in the Mammals collection of Museo de La Plata (MLP 1.I.03.11, MLP 1.I.03.12, MLP 1.I.03.13), La Plata, Buenos Aires Province, Argentina.

Prevalence and mean intensity of the helminths were calculated following Bush et al. (1997). For the nematode $N$. brasiliensis and for the cestode $H$. diminuta we calculated the minimum intensity because of the difficulty in separating the individual parasites in the first case and the difficulty to extracting the whole parasites in the second case. We classified parasite species on basis of to their prevalence as: central if it was present in more than $2 / 3$ of the hosts, secondary if it was present between $1 / 3$ and $2 / 3$ of the hosts and satellite, if it was present in less than $1 / 3$ of the hosts (Bush \& Holmes, 1986).

We compared the body mass and age of parasitized and non- parasitized hosts by means of T- test of independent samples or U-Mann Whitney test depending if the data fulfilled the assumptions of normality and covariance (Zar, 1996). To evaluate if the intensity of infection increases along the life of hosts, we studied the association between the mean intensity and the age of individuals by means of a Spearman correlation (Zar, 1996). We compared the inten- sity of infection among seasons by means of a KruskallWallis test (Zar, 1996).

We studied the existence of associations between the infections with different nematode species analyzing the frequency of co-occurrence of two species in the same individual by means of Chi-square test (Yates' correction) (Zar, 1996) and Forbes Index (Dice 1945) for determining if the frequency of coexistence was lower (Forbes $<1$ ) or higher than expected (Forbes $>1$ ) by chance.

\section{Results}

A total of $72 R$. norvegicus were captured during the study period. Nematodes were found in the intestine or caecum rectum of the $74 \%$ of rats, and cestodes were found along all the intestine of the $28 \%$ of rats. Nematodes include $H$. spumosa; S. muris and N. brasiliensis; whereas only one species of the cestode, $H$. diminuta, was found.

$H$. spumosa was a central species with a prevalence of $66 \%$, whereas $H$. diminuta, $S$. muris and $N$. brasiliensis were satellite species, with a prevalence of $28 \%, 14 \%$ and $11 \%$ respectively. There were two rats infected with three species of nematodes simultaneously.

H. spumosa was localized in the lower intestine and/or caecum-rectum. Body mass and age of rats parasitized (P) with $H$. spumosa were significantly higher than those of not parasitized (NP) $\left(\right.$ BODY MASS: mean $_{\mathrm{P}}=301 \mathrm{~g}$; $\mathrm{Sd}_{\mathrm{P}}=$ 94.7 and mean $_{\mathrm{NP}}=212 \mathrm{~g} ; \mathrm{Sd}_{\mathrm{NP}}=112.1$; respectively; $\mathrm{t}_{74}=$ 3.71; $\mathrm{p}<0.001$; $\underline{\mathrm{AGE}}:$ median $_{\mathrm{P}}=6$ months; $\mathrm{Q} 1=3.9 ; \mathrm{Q} 3=$ 7.1 and median $_{\mathrm{NP}}=4$ months, $\mathrm{Q} 1=1.8 ; \mathrm{Q} 3=5.8$, respectively; $\mathrm{U}_{73}=463 ; \mathrm{p}=0.02$; Fig. 1a). There was a significant positive association between intensity of infection (mean intensity $=33.74 ; \mathrm{Sd}=53.15$ ) with $H$. spumosa and the age of rats $\left(r_{s}=0.34 ; p<0.05\right)$. Mean intensity varied among seasons $\left(\mathrm{H}_{7,40}=14.56 ; \mathrm{p}=0.012\right)$, being greater in Summer than Autumn $2000\left(\mathrm{H}_{1,28}=4.81 ; \mathrm{p}=0.028\right)$ and Winter $2001\left(\mathrm{H}_{1,18} ; \mathrm{p}=0.001\right)$, and marginally greater than Spring $\left(\mathrm{H}_{1,18}=3.30 ; \mathrm{p}=0.069\right)$.

Nippostrongylus brasiliensis was localized in the upper intestine. There were no differences in body mass and age between rats infected and not infected (BODY MASS: $\operatorname{mean}_{\mathrm{P}}=216 \mathrm{~g}, \mathrm{Sd}_{\mathrm{P}}=134.1$ and $\operatorname{mean}_{\mathrm{NP}}=275 \mathrm{~g} ; \mathrm{Sd}_{\mathrm{NP}}=$ 104.8 , respectively; $\mathrm{t}_{74}=1.59 ; \mathrm{p}=0.114$; $\mathrm{AGE}_{\text {: }}$ median $_{\mathrm{P}}=$ 3 months; $\mathrm{Q} 1=1.8 ; \mathrm{Q} 3=5.8$ and median $_{\mathrm{NP}}=5.2$ months, $\mathrm{Q} 1=3.4 ; \mathrm{Q} 3=7.1$, respectively; $\mathrm{U}_{73}=207$; $\mathrm{p}=0.07$; Fig. 1b). Mean intensity of infection with $N$. brasiliensis (higher than 200 specimens) was higher in Autumn than in the other seasons, but the statistical analysis was not carried out because of the low number of individuals parasitized (Autumn 2000: 6, Winter 2000: 1, Spring 2000: 0, Summer 2000 - 2001: 2, Autumn 2001:0 and Winter 2001: 1). Syphacia muris was detected in the lower intestine and/or caecum-rectum of the host in Autumn. Body mass and age did not differ between rats infected and not infected with this parasite $\left(\underline{B O D Y}\right.$ MASS $:$ mean $_{\mathrm{P}}=276 \mathrm{~g}, \mathrm{Sd}_{\mathrm{P}}=$ 97.1; mean $_{\mathrm{NP}}=266 \mathrm{~g} ; \mathrm{Sd}_{\mathrm{NP}}=111.8$, respectively; $\mathrm{t}_{74}=0.2$; $\mathrm{p}=0.83 ; \underline{\mathrm{AGE}}$ : median $_{\mathrm{P}}=3.5$ months; $\mathrm{Q} 1=3.1 ; \mathrm{Q} 3=5.6$; median $_{\mathrm{NP}}=5$ months, $\mathrm{Q} 1=3.3 ; \mathrm{Q} 3=7.1$; respectively; $\mathrm{U}_{73}$ 

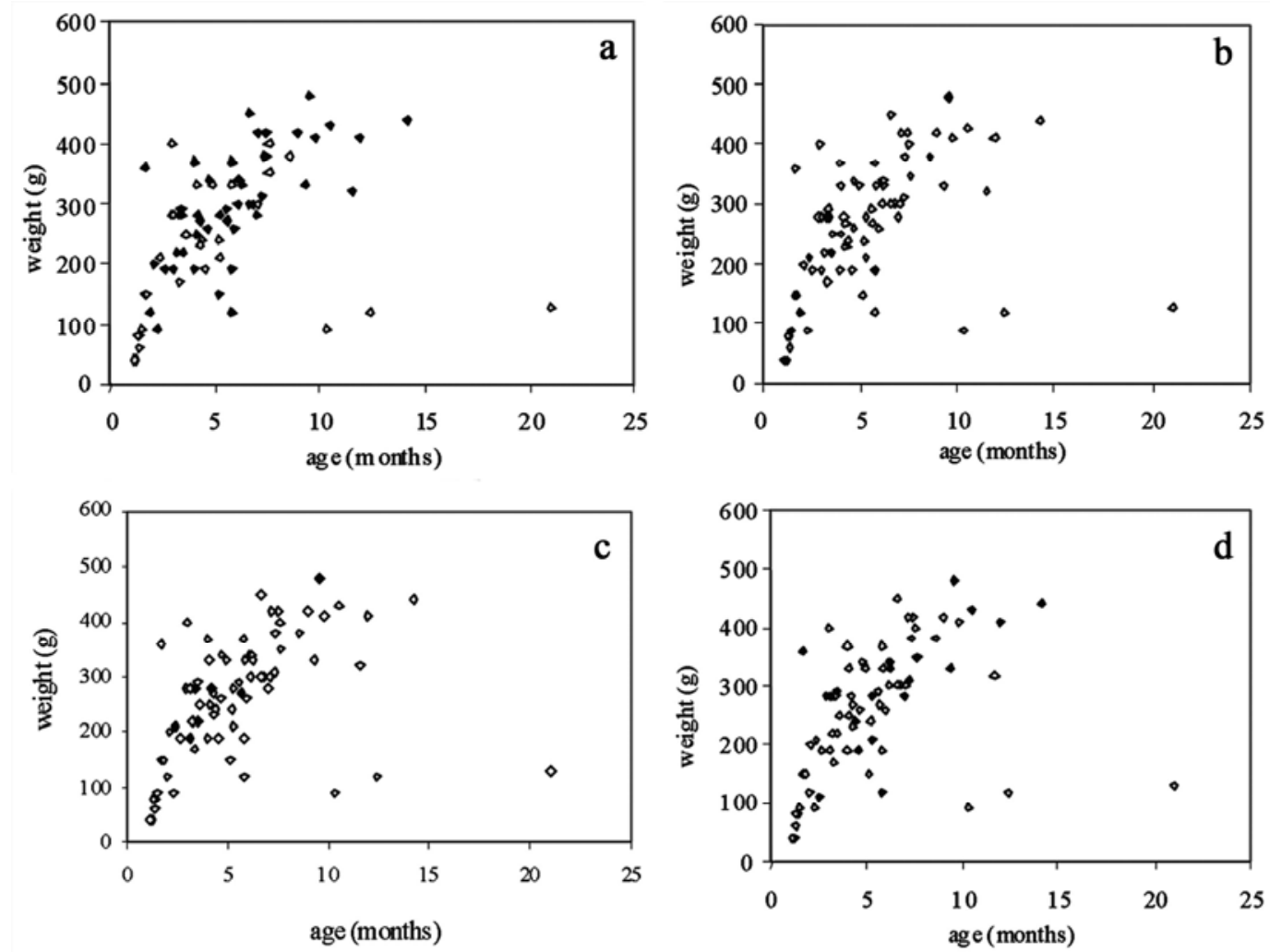

Fig. 1. Body mass and age of parasitized (dotted diamond) and no parasitized rats (open diamond) with: a) Heterakis spumosa b) Nippostrongylus brasiliensis, c) Syphacia muris and d) Hymenolepis diminuta.

$=188 ; \mathrm{p}=0.36 ;$ Fig. $1 \mathrm{c})$. There was no significant association between intensity of infection with $S$. muris (mean intensity $=74.28 ; \mathrm{S}=69.07)$ and host age $\left(\mathrm{r}_{\mathrm{s}}=\right.$ $0.50 ; \mathrm{p}=0.25)$.

Rats parasitized with $H$. diminuta were older than nonparasitized ones $\left(\right.$ median $_{\mathrm{P}}=6$ months; Q1 $=4.4 ; \mathrm{Q} 3=9.3$ and median $_{\mathrm{NP}}=4$ months, Q1 $=2.9 ; \mathrm{Q} 3=6.6$, respectively; $U_{73}=371 ; p=0.04$; Fig. $1 d$ ), and there was a trend to have a higher body mass, but differences were not significant $\left(\right.$ mean $_{\mathrm{P}}=304 \mathrm{~g}, \mathrm{Sd}_{\mathrm{P}}=101.1$ and mean $_{\mathrm{NP}}=252 \mathrm{~g} ; \mathrm{Sd}_{\mathrm{NP}}$ $=111.55$, respectively; $\left.\mathrm{t}_{74}=1.79 ; \mathrm{p}=0.07\right)$. H. diminuta was detected in all the seasons studied with a mean intensity of 3.10 per individual $(\mathrm{Sd}=3.47)$.

In relation with the association among helminths, we observed that the infection with $S$. muris and $N$. brasiliensis was independent $\left(X^{2}=0.23 ; \mathrm{p}=0.63\right)$ and with $H$. spumosa and $S$. muris was also independent in spite of both last species were located in the same portion of the intestine $\left(X^{2}=0.23 ; \mathrm{p}=0.63\right)$. To difference, H. spumosa and $N$. brasiliensis were negatively associated, and they were found in different portions of the intestine $\left(X^{2}=7.61 ; \mathrm{p}=\right.$ 0.006 Forbes $=0.3125$ ).

\section{Discussion}

The endoparasite community of brown rats that inhabiting poultry farms of Buenos Aires was characterized by four species, being $H$. spumosa a central species, and N. brasiliensis, $S$. muris and $H$. diminuta were satellite species.
As previously noted by Chaunmin et al. (1996) and Stojcevic et al. (2004), the parasitic infection do not affect rat survival and growth. The parasitized individuals were equal or higher and old, than non-parasitized. Additionally, the positive correlation between age of the rats and parasite intensity suggests that number of parasites was accumulated during the life of rats, and do not produce mortality of the individuals, being host and parasite adapted to each other (Anderson, 2000).

The seasonal variation in the intensity of infection depended on both the characteristics of the hosts and the climatic factors. The maximum intensity of infection for $H$. spumosa was occurred in summer, in agreement with the greater availability of earthworms that would be important in the transmission of this nematode (Anderson, 2000). On the other hand, rats had a maximum intensity of $N$. brasiliensis in Autumn. This would be possible because in this season occurs the greater precipitation in the study area, and $N$. brasiliensis needs humidity for its survival because it does not tolerate desiccation. The infection with $S$. muris only in Autumn, do not have a clear explanation. The transmission occurs by means of retroinfection and oral infection (direct cycle), so it would not be result of climatic factors. Additionally, it is not related to the size or age of the host. The infection would be result of rat behaviour such us grooming activities (Anderson, 2000).

The negative association between $H$. spumosa and N. brasiliensis may be the result of competition between them, or the different times of the year when the infection occurs. 
However, the first alternative is less probable because these species are located in different parts of the host, minimizing the interaction.

Among the helminth species detected, $H$. diminuta has importance to human health, due to produced gastrointestinal malaise, weakness and lack of appetite. In addition, the frequent presence of insects in poultry farms, enhance the probability that this parasite completes its cycle.

This study shows data of the community of helminths in rats in relationship with age and weight of their host, being one of the scarce studies made with those details.

\section{Acknowledgements}

We want to thank all the farmers who kindly let us work at their farms; to Graciela Navone, Celina Digiani and Cecilia Carballo who help in the determinations of helminths; to Emiliano Muschetto for help in the manipulations of intestines and the sources of financial support: University of Buenos Aires and National Council of Scientific and Technologic Research (CONICET) grants (UBACYT TX 58 and PIP 548/98).

\section{References}

Anderson, R. C., Chabaud, A. G., Willmott, S. (1974): CIH Keys to the nematode parasites of vertebrates. $68 \mathrm{pp}$. England, Commonwealth Agricultural Bureaux Press.

ANDERSON, R. C. (2000): Nematodes parasites of vertebrates. Their development and transmission., $2^{\text {nd }}$ Edition. 650 pp. CABI publishing, CAB international.

Bush, A. O., Holmes, J. C. (1986): Intestinal helminths of lesser scaup ducks: an interactive community. Can. J. Zool., 64: $142-152$

Bush, A. O., Lafferty, K. D., Lotz, J. M., Shostak, A.W. (1997): Parasitology meets ecology on its own terms: Margolis et al. revisted. J. Parasitol., 83: 575 - 583

CABrerA, R., MendozA, L. (2001): Heterakis spumosa Schneider, 1866 (Nematoda: Heterakidae) en Rattus norvegicus (Rodentia: Muridae) en Ica, Perú. Rev. peruana biol., 8 (1) (on line): http://sis bib.unmsm.edu.pe/bVrevistas/biologia/v08_nl/ heterakisrattus. Htm

Chaunmin, Y., Jiunjye, W., Juneder, L., YiPINE, C.,
ENGRIN, C. (1996): Parasitic infections among wild rats from two areas of Kaohsiung. Kao Hsiung J. Med. Science, 12: $145-149$

DiCE, L. (1945): Measures of the amount of ecologic association between species. Ecology, 26: 297 - 302

IANNACONE O. J., ALVARIÑO, L. F. (2002) : Helmintofauna de Rattus rattus (Linnaeus, 1758) y Rattus norvegicus (Berkenhout, 1769) (Rodentia: Muridae) en el distrito de San Juan de Lurigancho, Lima-Perú. Rev. Perú Med. Experimental y Salud Pública, 19: 136 - 141 Kataranovski, D., Kataranovski, M., SAVIC, I. R., CAKiC, P., Soldatovic, D., Matic, R. (1994): Morphometric and biochemical parameters as age indicators in the Norway Rat (Rattus norvegicus Berk, 1769). Acta Vet., 44: $371-378$

KhaliL, L. F., Jones, A., BrAy, R. A. (1994): Keys to the cestode parasite of vertebrates. $751 \mathrm{pp}$. Cambrige, University Press.

Pinto, R., Goncalves, L. Noronha, D. Gomes, D. C. (2001): Worm burdens in outbred and inbred laboratory rats with morphometric data on Syphacia muris (Yamaguti, 1935) Yamaguti, 1941 (Nematoda, Oxyuroidea). Mem. Inst. Oswaldo Cruz, 96: 133 - 136

SeOnG, J. K., HuH, S., LEE, J-S., OH, Y-S. (1995): Helminths in Rattus norvegicus captured in Chunchon, Korea. Korean J. Parasitol., 33: 235-237

StojCeVic, D., Mihaljevic, Z., Marinculic, A. (2004): Parasitological survey of rats in rural regions of Croatia. Vet. Med.(Praha), 49: $70-74$

Suriano, D. M., NAVOne, G. T. (1992): Systematics and ecology of Stilestrongylus freitasi Durette-Desset, 1968 and Hassalstrongylus multiovatus N. Sp. (Nematoda: Nippostrongylinae) from Akodon simulator (Cricetidae) in Tucumán (Argentina). Res. rev. Parasitol., 52: 51 - 55

SuRIANO, D. M., NAVONE, G. T. (1994): Three new species of the genus Trichuris Roederer, 1971 (Nematoda: Trichuridae) from cricetidae and octodontidae rodents in Argentina. Res. rev. Parasitol., 54: 39 - 46

WISNIVESK,Y. C. (2003): Ecología y epidemiología de las infecciones parasitarias. 398 pp. Argentina, Libro Universitario Regional Press.

ZAR, J. (1996): Biostatistical Analysis. $3^{\text {th }}$ Edition, 662 pp. United States of America, Prentice-Hall inc. 